\title{
Synthesis of functionalized 1-azabicycloheptanes/octanes/ nonanes - perspective building blocks for Medicinal Chemistry starting from proline
}

\section{S. Bondarenko, A. Fedorchenko, D. Volochnyuk, S. Ryabukhin}

\section{Introduction and Aim}

The shifting from "traditional" heteroaromatic design concepts in modern Medicinal Chemistry leads to increasing complexity of the synthesis of medicinally relevant molecules. One of the best way for solving this problem is to apply in the synthesis the robust well-known procedures and achieves complexity by using the sophisticated, commercially available (if it is possible), building blocks. Therefore, the interest in 3D-shaped building blocks with high fractions of $\mathrm{Fsp}^{3}$-hybridized carbon atoms continuously growth and now its synthesis becomes a part of the industry. Therefore, the procedures, which can effectively lead to the formation of targeted products in 10-100 grams scale become more and more demanded.

The effective multigram scale procedure for the synthesis of functionalized 1 -azabicycloheptanes/octanes/nonanes is proposed in our research. The method depends on using common organic reagents and simple reactions which highly increase its efficiency. On the other hand the products have been perspective building blocks for Medicinal Chemistry needs.

In this report, the synthetic methodology for the obtaining of functionalized 1-azabicycloheptanes/octanes/nonanes is validated and optimized. The advantages and scope and limitation of the procedure are determined and will be discussed.

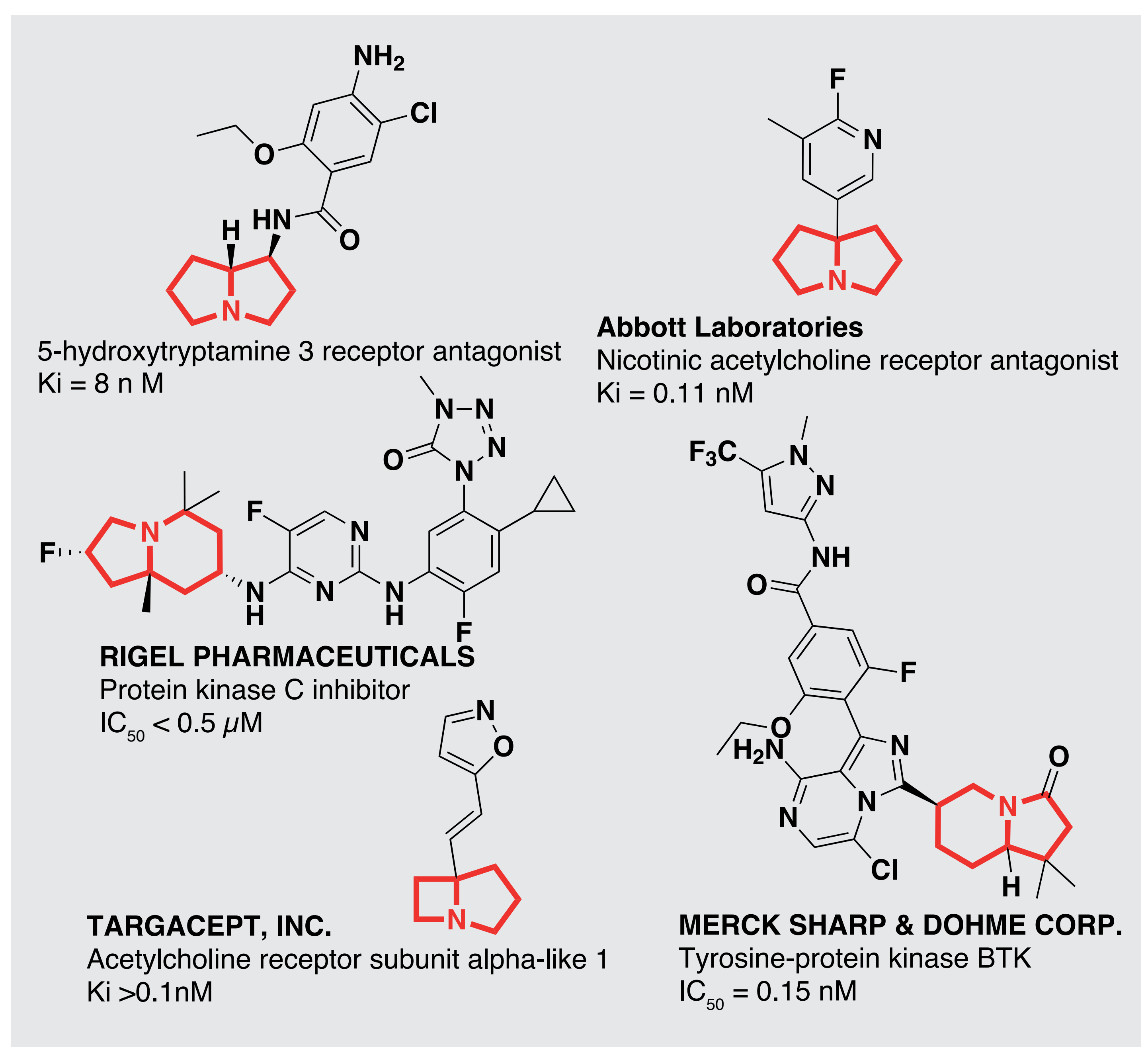

\section{Synthesis}
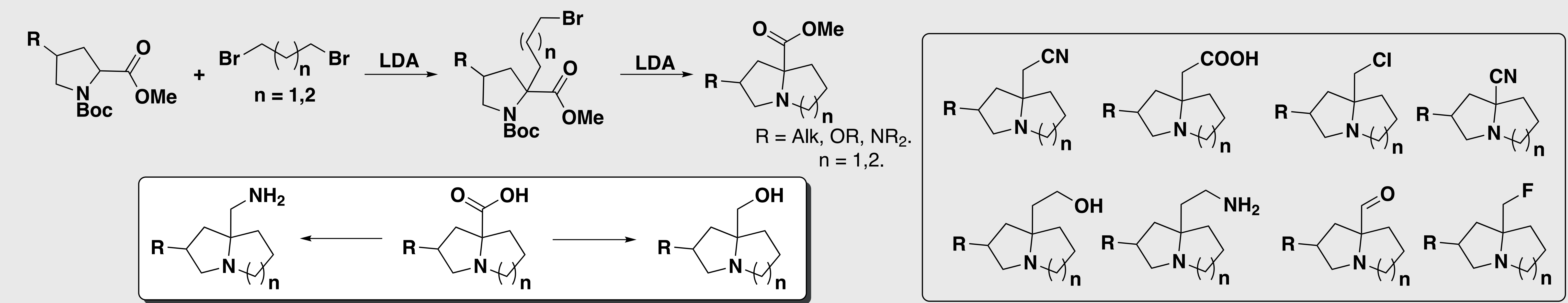

${ }_{\mathrm{H}}^{\mathrm{O}} \mathrm{OTtMS}_{\mathrm{Br}(\mathrm{CDA})_{2} \mathrm{OTMS}}^{\mathrm{O}}$

$\longrightarrow \mathrm{C}_{\mathrm{N}}^{\mathrm{NH}_{2}} \longrightarrow \mathrm{C}_{\mathrm{N}}^{\mathrm{OH}}$

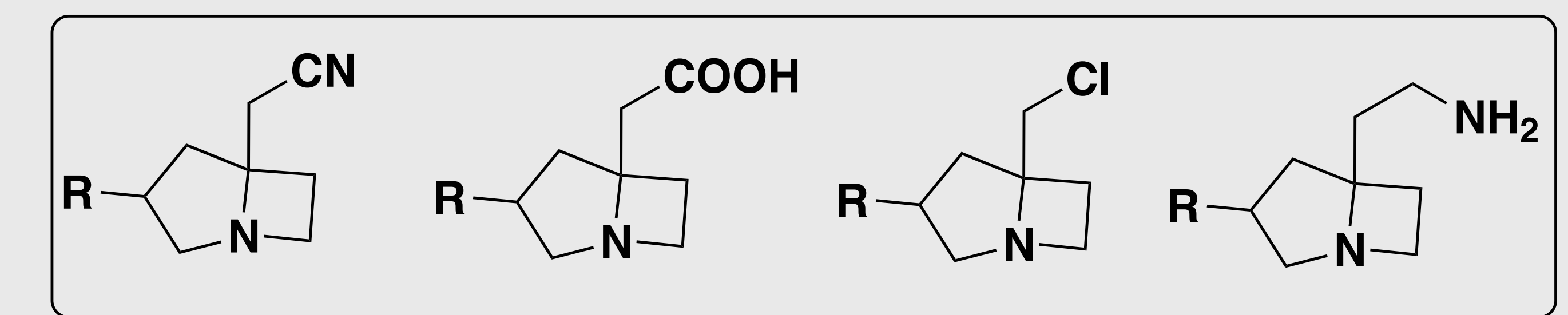

Results<smiles>O=C(O)C12CCCN1CCC2</smiles><smiles>OCC12CCCN1CCC2</smiles>

EN300-121038

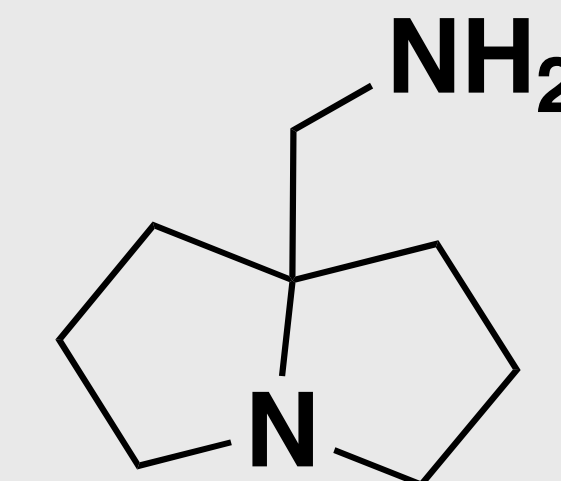

EN300-121037

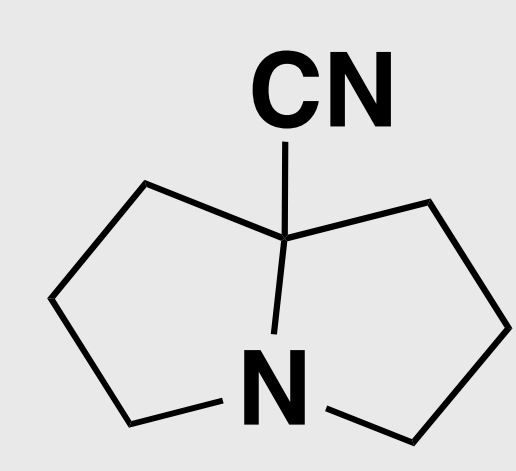

EN300-121036

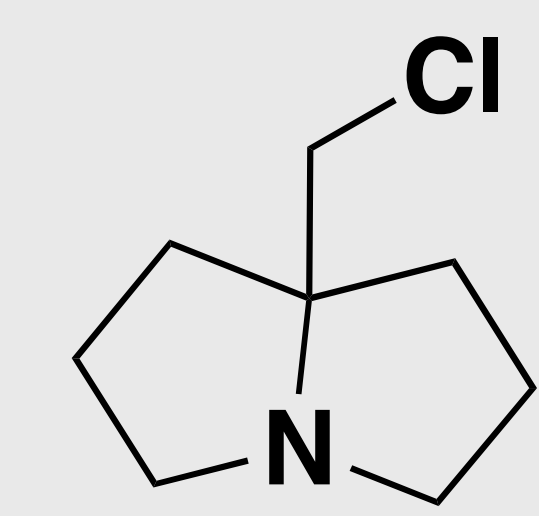

EN300-6491924

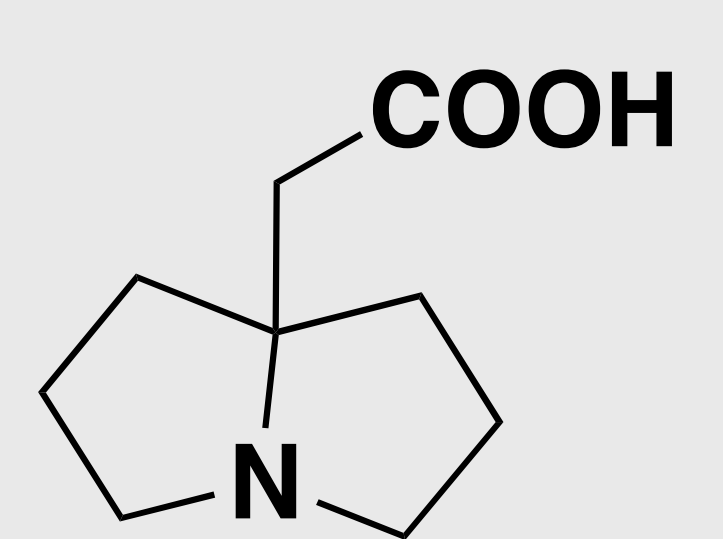

EN300-7420377

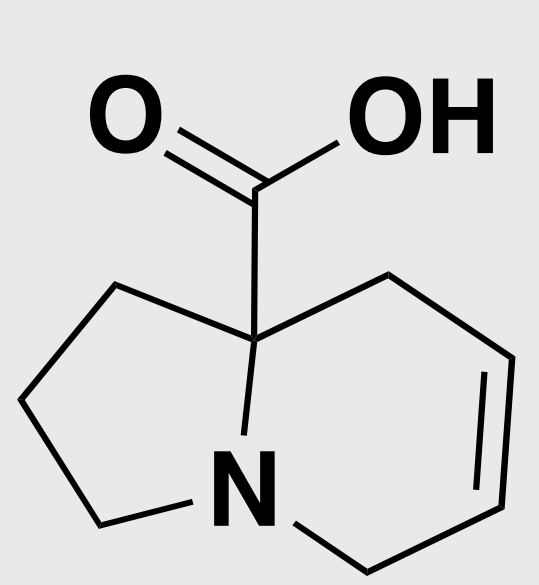

EN300-2009145

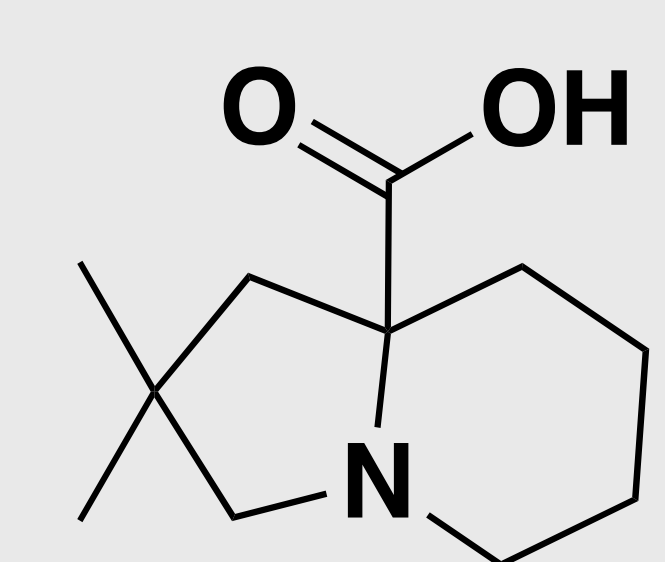

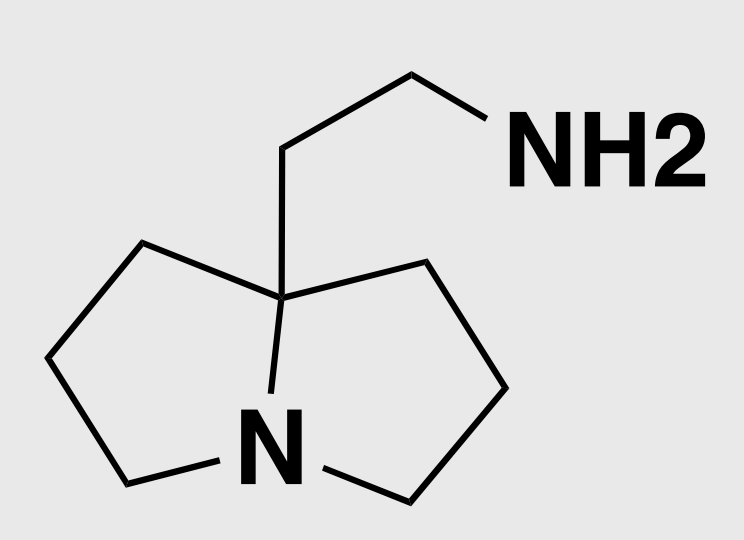

EN300-6769054<smiles>OCC12CC=CCN1CCC2</smiles>

EN300-26666742

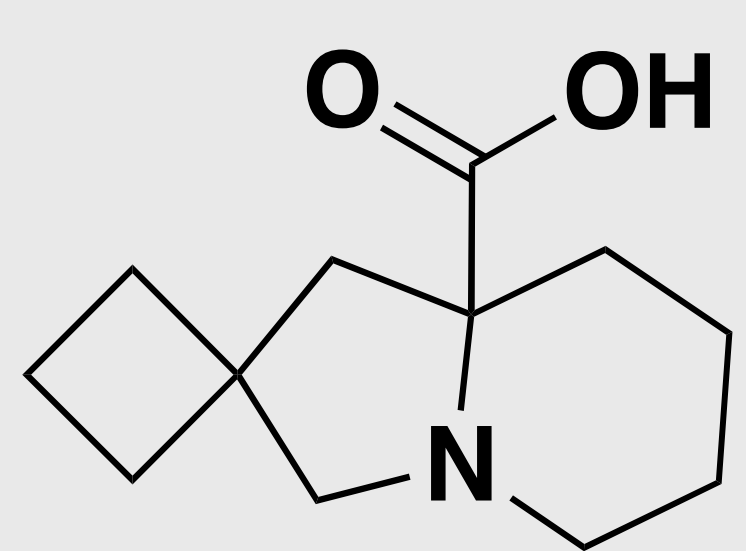<smiles>COC1CN2CCCC2(C(=O)O)C1</smiles>

EN300-2394701

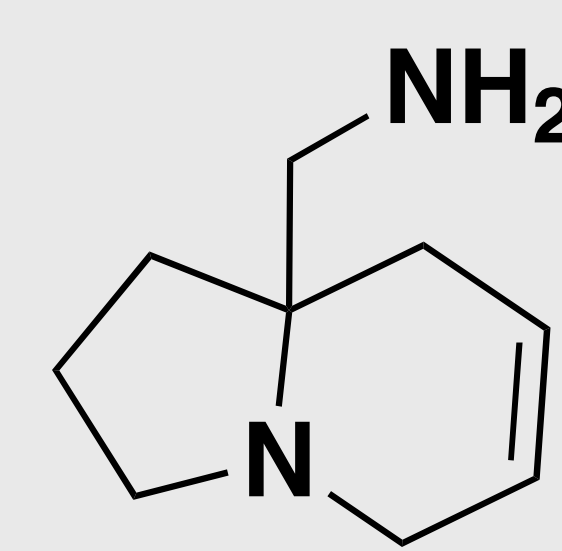

EN300-26666741

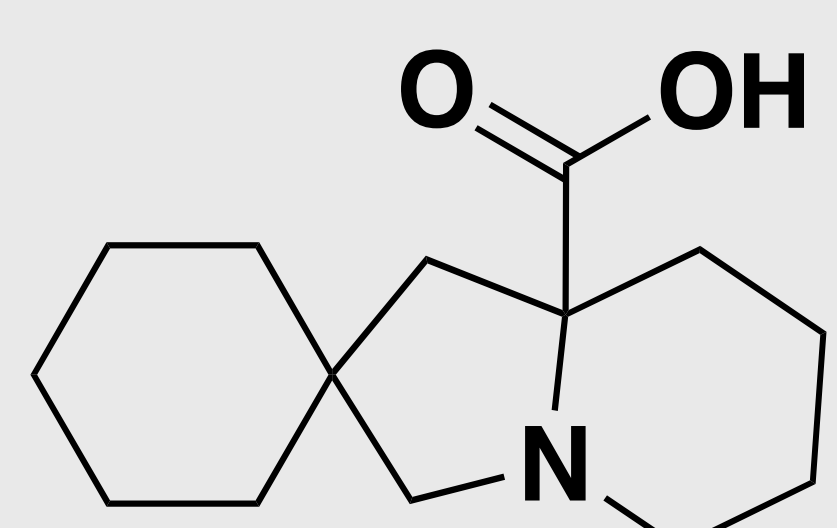

EN300-22986301 EN300-26666743 EN300-26666744 EN300-26666745 EN300-26666746 